\title{
Real-time cavity simulator-based low-level radio-frequency test bench and applications for accelerators
}

\author{
Feng Qiu, ${ }^{1,2, *}$ Shinichiro Michizono, ${ }^{1,2}$ Takako Miura, ${ }^{1,2}$ Toshihiro Matsumoto, ${ }^{1,2}$ \\ $\mathrm{Na} \mathrm{Liu},{ }^{2}$ and Sigit Basuki Wibowo ${ }^{3}$ \\ ${ }^{1}$ High Energy Accelerator Research Organization, 1-1 Oho, Tsukuba, Ibaraki 305-0801, Japan \\ ${ }^{2}$ The Graduate University for Advanced Studies, Shonan Village, Hayama, Kanagawa 240-0193, Japan \\ ${ }^{3}$ Department of Electrical Engineering and Information Technology, Universitas Gadjah Mada, \\ Grafika Street No. 2 Kampus UGM, Yogyakarta 55281, Indonesia
}

(Received 24 October 2017; published 16 March 2018)

\begin{abstract}
A Low-level radio-frequency (LLRF) control systems is required to regulate the rf field in the rf cavity used for beam acceleration. As the LLRF system is usually complex, testing of the basic functions or control algorithms of this system in real time and in advance of beam commissioning is strongly recommended. However, the equipment necessary to test the LLRF system, such as superconducting cavities and high-power rf sources, is very expensive; therefore, we have developed a field-programmable gate array (FPGA)-based cavity simulator as a substitute for real rf cavities. Digital models of the cavity and other rf systems are implemented in the FPGA. The main components include cavity baseband models for the fundamental and parasitic modes, a mechanical model of the Lorentz force detuning, and a model of the beam current. Furthermore, in our simulator, the disturbance model used to simulate the power-supply ripples and microphonics is also carefully considered. Based on the presented cavity simulator, we have established an LLRF system test bench that can be applied to different cavity operational conditions. The simulator performance has been verified by comparison with real cavities in KEK accelerators. In this paper, the development and implementation of this cavity simulator is presented first, and the LLRF test bench based on the presented simulator is constructed. The results are then compared with those for KEK accelerators. Finally, several LLRF applications of the cavity simulator are illustrated.
\end{abstract}

DOI: 10.1103/PhysRevAccelBeams.21.032003

\section{INTRODUCTION}

In accelerators, low-level radio-frequency (LLRF) systems are required to regulate the radio frequency (rf) fields in the rf cavities used for beam acceleration. In general, we must install and test these LLRF systems in advance of beam commissioning. In addition, for the examination of various of LLRF control algorithms during beam commissioning, functional testing is usually necessary. However, the conditions necessary for LLRF system testing, such as super-conducting environments, along with the required $\mathrm{rf}$ sources, are usually expensive and are not always available. This situations has motivated the authors to design realtime cavity simulators for LLRF system testing, which act as substitutes for the real platforms necessary to run an LLRF system.

\footnotetext{
qiufeng@ post.kek.jp
}

Published by the American Physical Society under the terms of the Creative Commons Attribution 4.0 International license. Further distribution of this work must maintain attribution to the author(s) and the published article's title, journal citation, and DOI.
Software-based (e.g., Simulink-based) state-space models of rf systems are common and have useful applications in the design process of an LLRF system [1]; however, it is difficult to operate the entire rf system in real time. An analog hardware-based cavity simulator has been developed to solve this problem [2]; however, the rf operational conditions within the analog components cannot be changed easily. In view of this situation, field-programmable gate array (FPGA)-based real-time cavity simulators have been developed [3-5]. Through use of those simulators, real-time behavior under LLRF control system operation can be obtained. However, those studies focused mainly on the fundamental mode of the cavity [3-5]. At present, multicell cavities are employed in many facilities such as the International Linear Collider (ILC), the compact energy recovery linac (cERL), and the superconducting test facility (STF) [6-12]. In such setups, the existence of the parasitic modes in the multi-cell cavities limits the loop gain of the LLRF feedback system. This behavior has motivated us to consider implementing not only the fundamental mode, but also the parasitic modes in the simulator. Furthermore, disturbances such as microphonics, power-supply ripples, and beam-loading limit the LLRF system performance, and 
valuable information can be obtained by incorporating these disturbances into the cavity simulator [13-15].

Here we present an FPGA-based cavity simulator considering both the fundamental mode and the parasitic modes; moreover, our simulator also integrates the common disturbances including microphonics, powersupply ripples, and beam-loading. This paper focuses on the design and implementation of the real-time cavity simulator and its applications. The organization is as follows. Section II briefly introduces the typical LLRF and cavity system incorporated in an accelerator. Section III describes the design of the cavity simulator. Section IV depicts the FPGA implementation of the simulator. Section V presents the cavity simulator-based LLRF test bench and compares its response with those for KEK accelerators. Finally, Sec. VI presents several successful applications of the presented simulator.

\section{LLRF SYSTEM}

A simplified block diagram of a typical LLRF control system is shown in Fig. 1. The pick-up rf signal (from the cavity) is down-converted to an intermediate frequency (IF) signal. After filtering by a low-pass filter, the filtered IF signal is sampled in the next stage by an analog-to-digital converter (ADC) and fed into an FPGA. The basebands in-phase and quadrature (I/Q) components are demodulated from the digitalized IF signal [16]. The I/Q signals are rotated by a rotation matrix to correct the loop phase, and then filtered by infinite impulse response (IIR) low-pass filters. Following comparison to their set values, the I/Q errors can be calculated. Then, the I/Q errors are regulated by a proportionalintegral (PI) feedback (FB) controller. The processed I/Q signals are added to their corresponding feed-forward (FF) models. The combinational signals are output to the I/Q modulator via digital-to-analog converters (DACs) to modulate the rf signal from the master oscillator. Finally, the LLRF feedback loop is closed by driving a high-power source, which drives the rf cavities.

As shown in Fig. 1, in order to test the LLRF system, rf devices (indicated by the red block) such as rf sources (e.g., a klystron) and rf cavities are required. However, these devices are not always available; thus, it is imperative that a real-time simulator is available as a substitute for real cavity and $\mathrm{rf}$ sources in the development process. On the other hand, the rf system is subjected to various disturbances. Typical sources include known disturbances such as highvoltage power-supply ripples, Lorentz force detuning (LFD), beam-loading, and unknown disturbances such as microphonics, master oscillator phase noise, and clock jitters [13-15]. These disturbances limit the performance of the LLRF system, therefore, valuable information can be

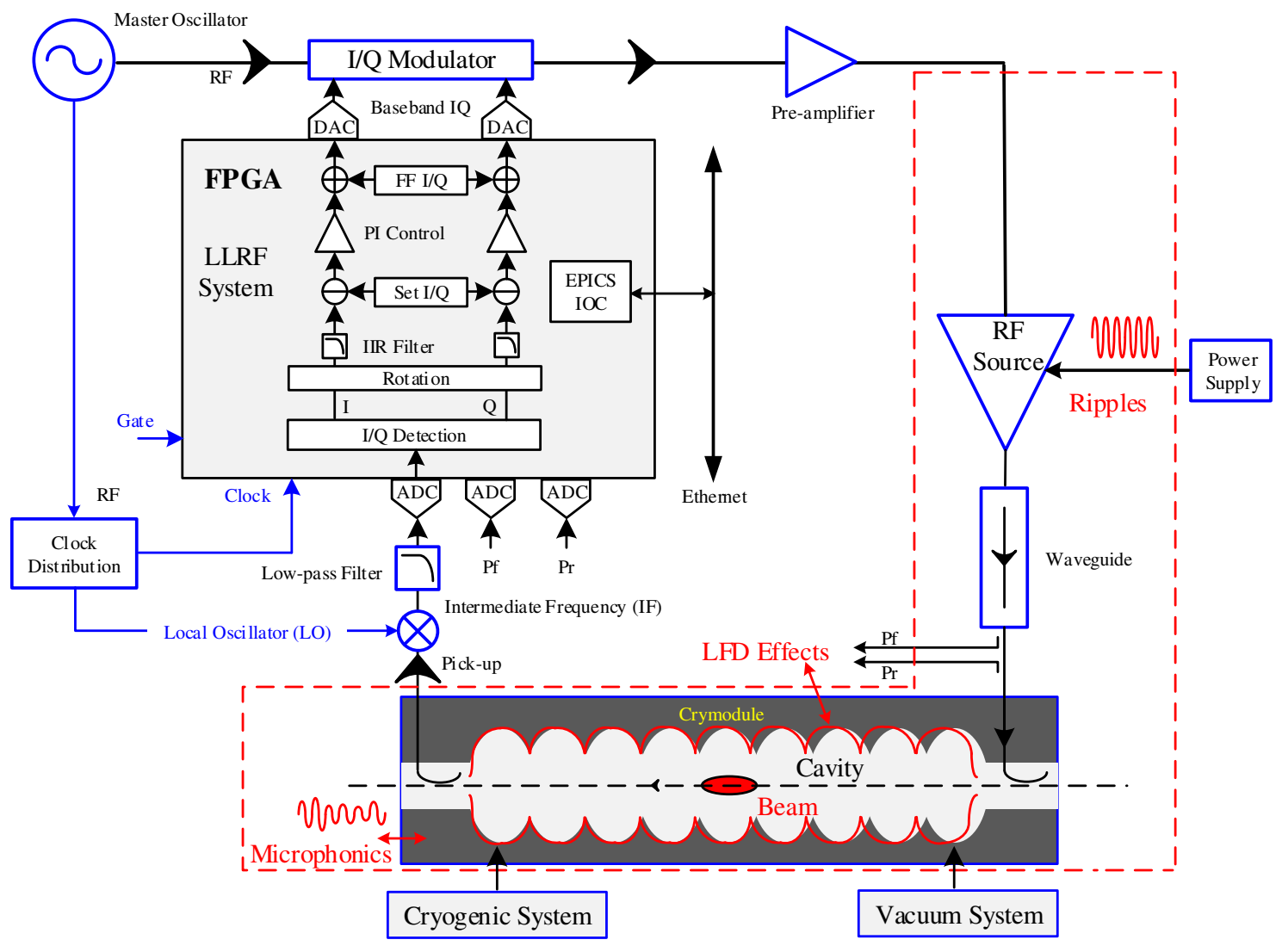

FIG. 1. Diagram of typical LLRF control system and rf cavity. 
obtained by incorporating the disturbances into the cavity simulator. In accordance with the above discussion, this paper presents the design and implementation of an FPGAbased real-time cavity simulator integrating both the rf cavity models and the rf disturbance models.

\section{CAVITY SIMULATOR DESIGN}

Figure 2 shows a functional block diagram of the cavity simulator. The simulator is fundamentally designed for superconducting cavities; however, the normal conducting cavity is also supported through selection of an appropriate coupling factor $\beta$. As the nine-cell cavity has become popular in many superconducting facilities such as cERL and STF at KEK $[6,12]$, in our design, we consider both the fundamental $\pi$ mode and the closest passband mode, i.e., the $8 \pi / 9$ mode [17]. In addition, we model the rf power source and the group delay. Furthermore, we incorporate the common sources of $\mathrm{rf}$ disturbance, such as powersupply ripples, microphonics, and beam-loading, in the simulator. We discuss the design and implementation of each component below.

\section{A. Cavity and mechanical model}

The rf cavity system can be modeled by combining an electrical model with a mechanical model $[3,5,16]$. Here, the electrical model, which describes the cavity baseband behavior, is based on the analysis by Schilcher [16]. The main idea is to represent the cavity by a resonant (LCR) circuit coupled to a waveguide driven by a klystron. According to Ref. [16], the cavity differential equation can be expressed by

$$
\frac{d \vec{V}_{c}}{d t}+\left(\omega_{1 / 2}-j \Delta \omega\right) \vec{V}_{c}=\omega_{1 / 2} \vec{u}
$$

and

$$
\vec{u}=\frac{2 \beta}{\beta+1} \vec{V}_{f}+R_{L} \vec{I}_{b}
$$

Here, the phasors $\vec{V}_{c}, \vec{V}_{f}$, and $\vec{I}_{b}$ represent the cavity voltage, cavity incident voltage, and the Fourier component of the beam current (at the operating frequency), respectively.

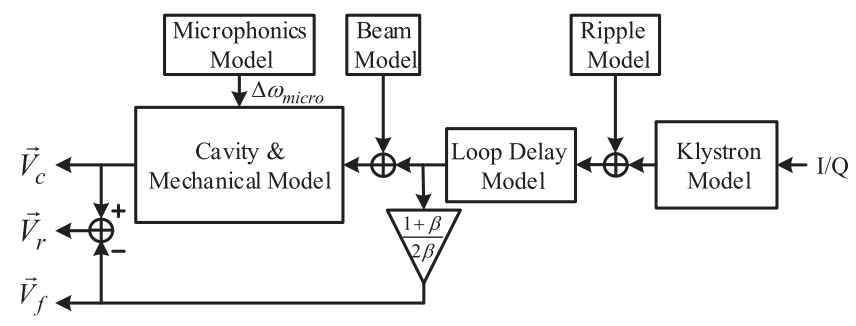

FIG. 2. Cavity simulator structure. Both the cavity model and the disturbance model are incorporated in the simulator.
The quantity $\omega_{1 / 2}$ is the cavity half bandwidth and the $\Delta \omega$ the cavity detuning. The parameter $\beta$ is the coupling factor and $R_{L}$ is the loaded resistance.

In the absence of the beam current, the cavity incident voltage $\vec{V}_{f}$ is given by

$$
\vec{V}_{f}=\frac{1+\beta}{2 \beta} \vec{u} .
$$

The reflected voltage $\vec{V}_{r}$ (as shown in Fig. 2), is easily calculated from

$$
\vec{V}_{r}=\vec{V}_{c}-\vec{V}_{f} .
$$

The state space formalism of (1) is given by

$$
\begin{aligned}
\frac{d}{d t}\left(\begin{array}{l}
V_{c, r} \\
V_{c, i}
\end{array}\right)= & \left(\begin{array}{cc}
-\omega_{1 / 2} & -\Delta \omega \\
\Delta \omega & -\omega_{1 / 2}
\end{array}\right)\left(\begin{array}{l}
V_{c, r} \\
V_{c, i}
\end{array}\right) \\
& +\left(\begin{array}{cc}
\omega_{1 / 2} & 0 \\
0 & \omega_{1 / 2}
\end{array}\right)\left(\begin{array}{l}
u_{r} \\
u_{i}
\end{array}\right) .
\end{aligned}
$$

Here, $V_{c, r}$ and $V_{c, i}$ represent the real and imaginary parts of the complex quantity $\vec{V}_{c}$, corresponding to the I and Q components of the cavity voltage, respectively.

In the case of a multicell cavity such as a nine-cell cavity, the differential equation for each passband mode is given by

$$
\frac{d \vec{V}_{c, n \pi / 9}}{d t}+\left(\omega_{1 / 2, n \pi / 9}-j \Delta \omega_{n \pi / 9}\right) \vec{V}_{c, n \pi / 9}=\omega_{1 / 2} \vec{u},
$$

where $\omega_{1 / 2, n \pi / 9}$ and $\Delta \omega_{n \pi / 9}$ represent the half bandwidth and detuning of the $n \pi / 9$ mode, respectively. The overall cavity voltage is then given by the superposition of all the passband modes, where [17]

$$
\vec{V}_{c, \text { total }}=\sum_{n=1}^{9}(-1)^{n-1} V_{c, n \pi / 9} .
$$

If the closest passband mode only is considered, i.e., the $8 \pi / 9$ mode, the cavity voltage is then expressed by

$$
\vec{V}_{c, \text { total }}=\vec{V}_{c}-\vec{V}_{c, 8 \pi / 9} .
$$

In order to implement (6) in the FPGA, we must convert the models in continuous-time form to discrete-time form. The discrete-time form of (1) is required in the FPGA. References [3,5] give the cavity differential equation in discrete-time form (discrete-I) as

$$
\begin{aligned}
{\left[\begin{array}{l}
V_{c, r}(n) \\
V_{c, i}(n)
\end{array}\right]=} & {\left[\begin{array}{cc}
1-T_{s} \omega_{1 / 2} & -T_{s} \Delta \omega \\
T_{s} \Delta \omega & 1-T_{s} \omega_{1 / 2}
\end{array}\right]\left[\begin{array}{c}
V_{c, r}(n-1) \\
V_{c, i}(n-1)
\end{array}\right] } \\
& +\left[\begin{array}{cc}
T_{s} \omega_{1 / 2} & 0 \\
0 & T_{s} \omega_{1 / 2}
\end{array}\right]\left[\begin{array}{l}
u_{r}(n-1) \\
u_{i}(n-1)
\end{array}\right] .
\end{aligned}
$$

Where, the quantity $T_{s}$ represents the sampling period of the digital board that implements the cavity difference 

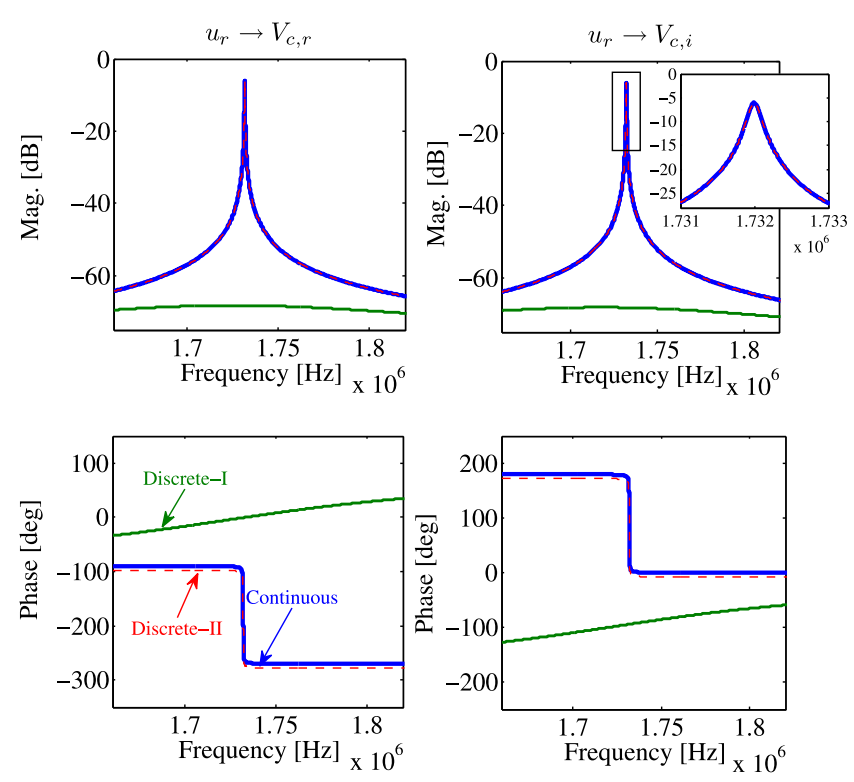

FIG. 3. Comparison of bode plots from $u_{r}$ to $V_{c, r}$ and $V_{c, i}$. Left: $u_{r} \rightarrow V_{c, r}$, right: $u_{r} \rightarrow V_{c, i}$. The bode plot of the continuous-time (blue) and discrete-I (green) cases are very different; however, the discrete-II (red) case is very similar to the continuous-time case.

equation. This equation works well for the fundamental mode ( $\pi$ mode), in which the detuning $|\Delta \omega|$ is far less than $\frac{1}{T_{s}}$. However, in the case of the parasitic mode of a multicell cavity, in which the values of $\Delta \omega_{8 \pi / 9}$ and $\frac{1}{T_{s}}$ are comparable, the difference equation in discrete-I described by (9) above may generate problems.

We assume that the value of the $\Delta \omega_{8 \pi / 9}$ is $1.732 \times 10^{6}$. $2 \pi \mathrm{rad} / \mathrm{s}$ (the detuning of the $8 \pi / 9$ mode of the nine-cell cavities in the main linac of the KEK-cERL) and that the $T_{s}$ is approximately $81.25 \mathrm{MHz}$. The corresponding bode plots from the input $\vec{u}$ to the output $\vec{V}_{c}$ of the continuous-time case in (5) and the discrete-time case in (9) are shown in Fig. 3. The bode plots in blue indicate the continuous-time case, whereas the green plots represent the discrete-I case. Extremely large deviations can be observed in the figure.

Let us return to the original cavity differential equation in (1), with a transfer function given by

$$
H_{\mathrm{cav}}(s)=\frac{\omega_{1 / 2}}{s+\omega_{1 / 2}-j \Delta \omega} \text {. }
$$

As the pole in the Laplace transform (10) is $s_{p}=-\omega_{1 / 2}+j \Delta \omega$, the pole in the Z-transform can be defined by

$$
z_{p}=e^{s_{p} T_{s}}=e^{-\omega_{1 / 2} T_{s}+j \Delta \omega T_{s}}
$$

Then, the transfer function in discrete-time form is given by

$$
H_{\mathrm{cav}}(z)=\frac{b}{z-z_{p}} .
$$

Note that the gain of $H_{\text {cav }}(s)$ is $0 \mathrm{~dB}$ at $s=j \Delta \omega$, meaning that $H_{\text {cav }}(z)$ should also be $0 \mathrm{~dB}$ at $z=e^{j \Delta \omega T_{s}}$; hence, we obtain $b=1-e^{-T_{s} \omega_{1 / 2}}$. Finally, we have

$$
H_{\mathrm{cav}}(z)=\frac{\left(1-e^{-T_{s} \omega_{1 / 2}}\right) z^{-1}}{1-\left(e^{-\omega_{1 / 2} T_{s}+j \Delta \omega T}\right) z^{-1}} .
$$

From (13), the discrete-time equation (discrete-II) is expressed by

$$
\begin{aligned}
{\left[\begin{array}{l}
V_{c, r}(n) \\
V_{c, i}(n)
\end{array}\right]=} & \mathbf{A}\left[\begin{array}{c}
V_{c, r}(n-1) \\
V_{c, i}(n-1)
\end{array}\right] \\
& +\left[\begin{array}{cc}
1-e^{-T_{s} \omega_{1 / 2}} & 0 \\
0 & 1-e^{-T_{s} \omega_{1 / 2}}
\end{array}\right]\left[\begin{array}{l}
u_{r}(n-1) \\
u_{i}(n-1)
\end{array}\right]
\end{aligned}
$$

where

$$
\mathbf{A}=\left[\begin{array}{cc}
e^{-T_{s} \omega_{1 / 2}} \cos \left(T_{s} \Delta \omega\right) & -e^{-T_{s} \omega_{1 / 2}} \sin \left(T_{s} \Delta \omega\right) \\
e^{-T_{s} \omega_{1 / 2}} \sin \left(T_{s} \Delta \omega\right) & e^{-T_{s} \omega_{1 / 2}} \cos \left(T_{s} \Delta \omega\right)
\end{array}\right] .
$$

Figure 3 also compares the bode plots of this discrete-II case (red) with the continuous case (blue). It is clearly apparent that these two cases are in good agreement in terms of their bode plots. The small phase lag in the discrete-II case is caused by the clock latency in (14). It should be mentioned that another discrete-time form of (5), similar with discrete-II, was derived in [16].

Note that, when $T_{s} \omega_{1 / 2} \rightarrow 0$ and $T_{s} \Delta \omega \rightarrow 0$, we have

$$
\left\{\begin{array}{l}
e^{-T_{s} \omega_{1 / 2}} \rightarrow 1-T_{s} \omega_{1 / 2} \\
1-e^{-T_{s} \omega_{1 / 2}} \rightarrow T_{s} \omega_{1 / 2} \\
\cos \left(T_{s} \Delta \omega\right) \rightarrow 1 \\
\sin \left(T_{s} \Delta \omega\right) \rightarrow T_{s} \Delta \omega .
\end{array}\right.
$$

It is clear that (9) is actually a special case of (14).

In the pulse-mode operation, the cavity is deformed by the Lorentz force due to the large magnetic field and the wall current. As a result, several mechanical resonance modes are excited by the Lorentz force and $\Delta \omega$ is seriously disturbed. The dynamics of the LFD is described by a set of second-order differential equations [18]

$\frac{d^{2} \Delta \omega_{k}^{(\mathrm{acc})}}{d t^{2}}+\frac{\omega_{k}}{Q_{k}} \frac{d \Delta \omega_{k}^{(\mathrm{acc})}}{d t}+\omega_{k}^{2} \Delta \omega_{k}^{(\mathrm{acc})}=-\omega_{k}^{2} K_{k} E_{\mathrm{acc}}^{2}$,

where $\Delta \omega_{k}^{(\mathrm{acc})}$ is the detuning of the accelerating eigenmode due to the mechanical mode $k$. The quantity $E_{\text {acc }}$ represents the accelerating gradient of a cavity defined by [18] 


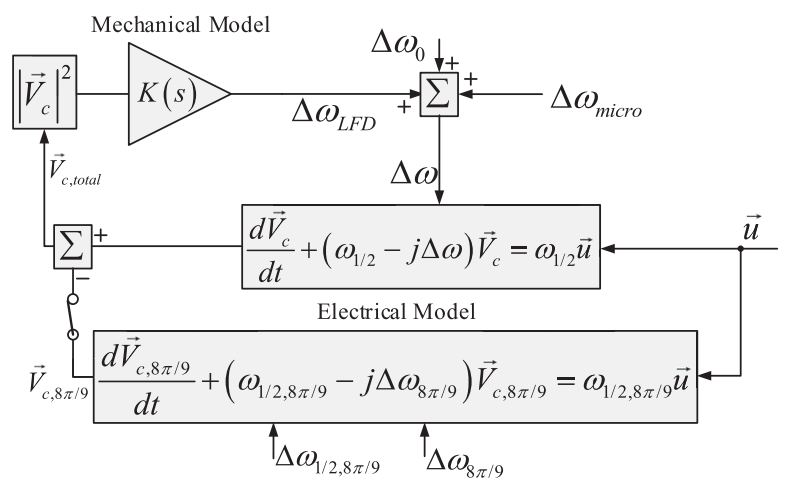

FIG. 4. Electrical and mechanical models of cavity system. Electro-mechanical interaction is considered here.

$$
E_{\text {acc }}:=\frac{V_{c}}{L}, \quad L=\text { cavity effective length. }
$$

The parameters $K_{k}, \omega_{k}$, and $Q_{k}$ represent the LFD constant, the resonance frequency, and the quality factor of the $k^{\text {th }}$ mechanical mode, respectively. Then, the overall LFD is given by

$$
\Delta \omega_{\mathrm{LFD}}=\sum_{k} \Delta \omega_{k}^{(\mathrm{acc})}
$$

The total detuning $\Delta \omega$ is the superposition of $\Delta \omega_{\mathrm{LFD}}$, the microphonics detuning $\Delta \omega_{\text {micro }}$, and the detuning offset $\Delta \omega_{0}$, as expressed by

$$
\Delta \omega=\Delta \omega_{\mathrm{LFD}}+\Delta \omega_{\text {micro }}+\Delta \omega_{0} .
$$

It is easy to transform the differential equation given in (17) to its transfer function form:

$$
H_{k}(s)=\frac{\Delta \omega_{k}(s)}{V_{c}^{2}(s)}=\frac{-\left(\frac{\omega_{k}}{L}\right)^{2} K_{k}}{s^{2}+\frac{\omega_{k}}{Q_{k}} s+\omega_{k}^{2}} .
$$

Then, the mechanical model $K(s)$ in Fig. 4 is

$$
K(s)=\frac{\Delta \omega_{\mathrm{LFD}}(s)}{V_{c}^{2}(s)}=\sum_{k} H_{k}(s) .
$$

Finally, the integrated electro-mechanical model of the cavity is constructed as shown in Fig. 4. The switch is adopted to make the model compatible with the single-cell cavities.

\section{B. Klystron model}

The klystron model in Fig. 2 is modeled by a first-order low-pass filter with a 3 -dB bandwidth $f_{\text {kly }}$ of several $\mathrm{MHz}$ depending on the particular klystron. In Ref. [19], the klystron nonlinearity is taken into account in the cavity simulator. However, because of the limitation of the FPGA resources, the klystron nonlinearity is not considered in our simulator. Here,

$$
H_{\mathrm{kly}}(s)=\frac{2 \pi f_{\mathrm{kly}}}{s+2 \pi f_{\mathrm{kly}}} .
$$

\section{Delay model}

In order to simulate the group delay $T_{d}$ introduced by the rf transmission lines (and other rf components), we insert a delay model. The loop delay model is described by

$$
H_{\text {delay }}(s)=e^{-T_{d} s} \text {. }
$$

\section{Disturbance model}

As mentioned above, the disturbances of the rf system are incorporated into our simulator. As the LFD is already contained in the mechanical model, we focus on the powersupply ripples, the microphonics, and the beam-loading only in the design of the disturbance model.

The power-supply ripples mainly influence the phase of the rf signal. According to Ref. [20], if the cathode voltage of the klystron has a ripple component of $\Delta V_{k} \sin \left(\omega_{r p} t\right)$, the klystron output is phase-modulated and can be expressed as

$$
y_{r p}=A \sin \left[\omega_{r f} t+D \frac{\Delta V_{k}}{V_{k}} \sin \left(\omega_{r p} t\right)\right],
$$

where $\omega_{r f}, \omega_{r p}, V_{k}$, and $D$ are the $\mathrm{rf}$ frequency, the ripple frequency, the cathode voltage, and the voltage fluctuation coefficient, respectively.

The microphonics is modeled by the superposition of a serials of sinusoidal signals $A_{m} \sin \left(\omega_{m} t+\phi_{m}\right)$ with different amplitude $A_{m}$, phase $\phi_{m}$, and frequency $\omega_{m}$ values [21-23]. The detuning caused by the microphonics is then expressed by

$$
\Delta \omega_{\text {micro }}=\sum_{m} A_{m} \sin \left(\omega_{m} t+\phi_{m}\right) .
$$

It should be mentioned that the second order response of the mechanical system need to be taken in to account in the microphonics model in principle. Since our simulator mainly focus on the field control problem, any second order model for microphonics is not available in the current version. For the further study such as microphonics detuning compensation in CW mode [21], the second order model need to be carefully considered in future works.

The beam current is actually part of the driven term $\vec{u}$ in (1). For the simulation, we added a beam table to the input of the cavity model. The beam model is activated after the beam trigger. 


\section{IMPLEMENTATION OF CAVITY SIMULATOR IN FPGA}

Before implementing the simulator in the FPGA, all the models must be transformed to discrete-time form. Here, we define our method for the continuous-time to discretetime conversion, excluding the cavity model, which has already been converted above. Block diagrams of the main models are also presented.

\section{A. Implementation of cavity model in FPGA}

The cavity differential equation in discrete form is given by (9) and (14), corresponding to discrete-I (for the fundamental mode) and discrete-II (for the parasitic mode), respectively. In the FPGA, $\vec{V}_{c}$ is then calculated by adding the outputs of the discrete-I and discrete-II cases. Figure 5 shows the structure of the cavity model. Note that the structures are identical for both the discrete-I and discrete-II cases. The differences are the values of the coefficients $a, b$, and $c$.

The mechanical mode $K(s)$ can be directly converted to its discrete-time form $K(z)$ by the Matlab function "c2d". The bilinear (Tustin) approximation method is adopted in this conversion. The model $K(z)$ is simplified as a highorder IIR filter (e.g., fourth order) with difference equation

$$
y(n)=\sum_{k=1}^{k=K} a_{k} y(n-k)+\sum_{m=0}^{m=M} b_{m} x(n-m) .
$$

where the sequences $x(n)$ and $y(n)$ represent the $\left|\vec{V}_{c}\right|^{2}$ and $\omega_{\text {LFD }}$ sequences, respectively.

\section{B. Implementation of klystron model in FPGA}

It is easy to transform $H_{\mathrm{kly}}(s)$ to its discrete form $H_{\mathrm{kly}}(z)$ by matching their poles. As the transfer function in (23) has a single pole $s_{p}=-2 \pi f_{\mathrm{kly}}$, the transfer function $H_{\mathrm{kly}}(z)$ has a pole at $z_{p}=e^{-2 \pi f_{\mathrm{kly}} T_{s}}$, and can be defined by

$$
H_{\mathrm{kly}}(z)=\frac{\left(1-e^{-2 \pi f_{\mathrm{kly}} T_{s}}\right) z^{-1}}{1-e^{-2 \pi f_{\mathrm{kly}} T_{s}} z^{-1}} .
$$

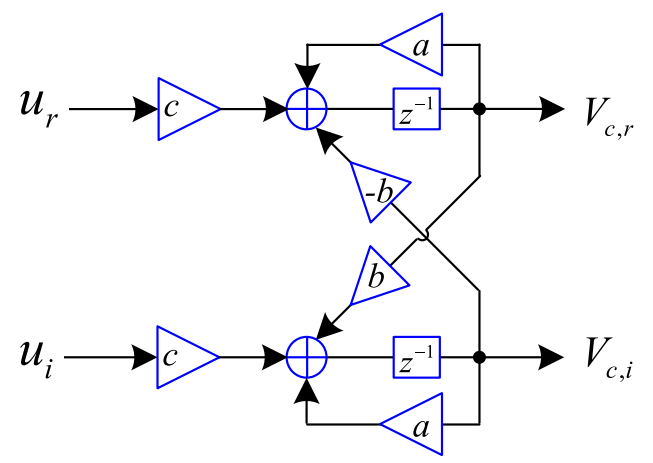

FIG. 5. Structure of cavity model in FPGA. The parameters $a$, $b$, and $c$ are selected based on difference equations (9) and (14).

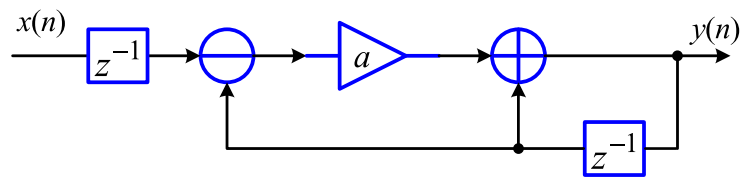

FIG. 6. Structure of klystron model. The parameter $a$ is equivalent to $1-e^{-2 \pi f_{\mathrm{kly}} T_{s}}$.

The corresponding difference equation is given by

$y(n)=e^{-2 \pi f_{\mathrm{kly}} T_{s}} y(n-1)+\left(1-e^{-2 \pi f_{\mathrm{kly}} T_{s}}\right) x(n-1)$,

where the $x(n)$ and $y(n)$ sequences represent the baseband klystron input and output (without ripples) signals, respectively.

In order to conserve the digital signal processing (DSP) resources in the FPGA, we convert (29) to

$y(n)=\left(1-e^{-2 \pi f_{\mathrm{kly}} T_{s}}\right)[x(n-1)-y(n-1)]+y(n-1)$.

The FPGA implementation of (30) is shown by Fig. 6.

\section{Implementation of delay model in FPGA}

A digital delay model $G_{T_{d}}(z)$ is applied to simulate the delay caused by the rf transmission line:

$$
G_{T_{d}}(z)=z^{-L}, \quad \text { and } \quad L=\left\lceil\frac{T_{d}}{T_{s}}\right\rceil,
$$

where the operator " $\rceil\rceil$ " indicates rounding of the elements inside the brackets to the nearest integers.

\section{Implementation of disturbance model}

The klystron output is usually phase-modulated by ripples, as shown in (26). If we define $\phi_{r p}=D \frac{\Delta V_{k}}{V_{k}} \sin \left(\omega_{r p} t\right)$, the output of the klystron with ripples becomes

$$
y=\sin \left(\omega_{r f} t+\theta+\phi_{r p}\right),
$$

with corresponding I/Q components $I_{r p}$ and $Q_{r p}$ given by $\left\{\begin{array}{l}I_{r p}=A \cos \left(\theta+\phi_{r p}\right)=A \cos \theta \cos \phi_{r p}-A \sin \theta \sin \phi_{r p}, \\ Q_{r p}=A \sin \left(\theta+\phi_{r p}\right)=A \sin \theta \cos \phi_{r p}+A \cos \theta \sin \phi_{r p} .\end{array}\right.$

Note that $I=A \cos \theta$ and $Q=A \sin \theta$ represent the IQ components of the klystron output without ripples. On the other hand, the value of $\left|\phi_{r p}\right|$ is usually less than 2 degrees (in the cERL, $\left|\phi_{r p}\right|$ is approximately 1.6 degrees); then, we have $\cos \phi_{r p} \approx 1$ and $\sin \phi_{r p} \approx \phi_{r p}$. Therefore, (33) is simplified to 


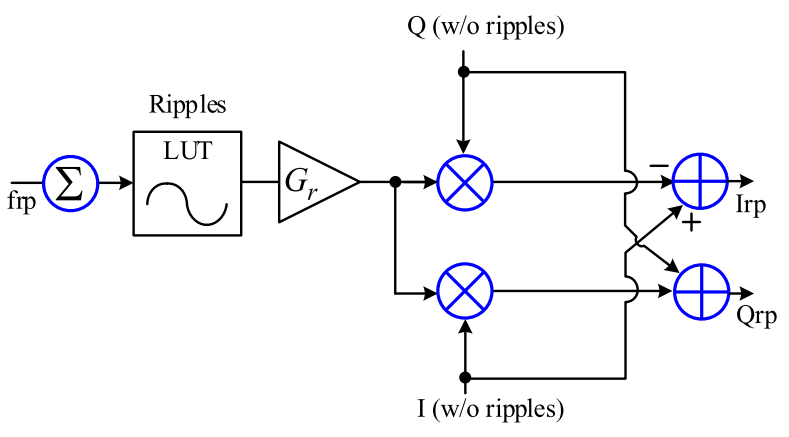

FIG. 7. Structure of ripple model in FPGA. The parameter "frp" in the figure represents the ripple frequency, and the ripple amplitude is scaled by the parameter " $G_{r}$ ".

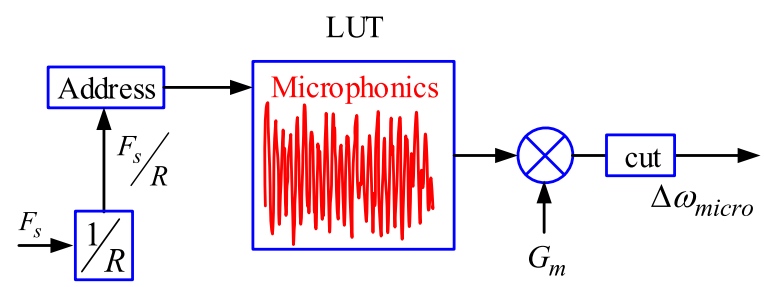

FIG. 8. Structure of microphonics model in FPGA. The measured microphonics data obtained from the real cavity data are prestored in the LUT. The parameter " $G_{m}$ " input port is used to control the amplitude of the microphonics.

$$
\left\{\begin{array}{l}
I_{r p}=I \cos \phi_{r p}-Q \sin \phi_{r p} \approx I-Q \cdot \phi_{r p} \\
Q_{r p}=Q \cos \phi_{r p}+I \sin \phi_{r p} \approx Q+I \cdot \phi_{r p} .
\end{array}\right.
$$

The structure of the ripple model in the FPGA is illustrated by Fig. 7. The input port "frp" in the figure regulates the ripple frequency. The ripple waveform $\sin \left(\omega_{r p} t\right)$ is saved in the lookup table (LUT) of Fig. 7. The ripple amplitude is controlled by another input port, labeled " $G_{r}$ ". After processing by (34), the IQ signal modulated by the power-supply ripples is output via the "I/Q out" ports.

The functional block of the microphonics is illustrated in Fig. 8. The measured microphonics data from the real cavities is stored in the LUT and then output after proper scaling.

The beam model works in both pulse-mode operation and burst-mode operation [12]. If the beam is triggered, a simulated beam signal with trapezoid profile is added to the input port of the cavity model (see Fig. 2). Parameters such as the beam current, beam phase, and beam width are adjustable.

\section{VERIFICATION AND CONFIRMATION}

To established the presented simulator-based LLRF test bench, two micro telecommunications computing architecture ( $\mu$ TCA) FPGA boards (see Fig. 9) equipped with four

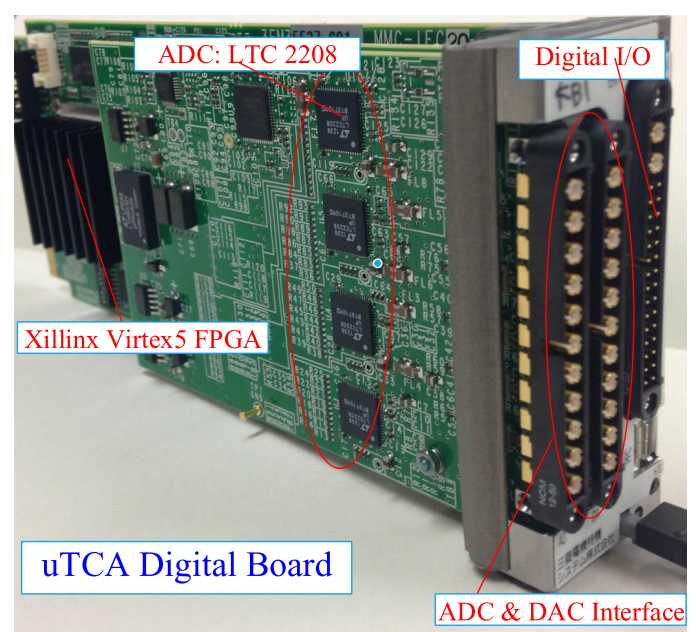

FIG. 9. MicroTCA FPGA board used performing digital algorithms in cavity simulator.

16-bit ADCs and DACs were utilized. As shown in Fig. 10, one board functions as the conventional LLRF system (FPGA \#2), while the other one is used as the presented simulator (FPGA \#1). The real and imaginary parts (I/Q components) of $\vec{V}_{c}$ are output to an IQ modulator via DAC1 and DAC2 of FPGA \#1. In DAC3 and DAC4, the cavity incident voltage $\left(\vec{V}_{f}\right)$ signal and cavity reflect signal $\left(\vec{V}_{r}\right)$, which are modulated by IF, are exported. After filtering, all of these signals are sampled by ADCs and fed into FPGA \#2, on which the LLRF control algorithm is performed. The IQ components in each signal are detected first, and the cavity signal is then processed and output to the IQ modulator to modulate the rf signal from the master oscillator. This rf signal is down-converted to the IF and then sampled by the ADC1 of the FPGA \#1. Finally, the discrete IF signal drives the cavity simulator. On these two FPGA boards, embedded Linux is installed in a Power-PC. The experimental physics and industrial control system (EPICS) is also installed for communication control, and each board acts as an EPICS input/output controller (IOC).

Table I compares the resources used in the cavity simulator and the available resources in the $\mu \mathrm{TCA}$ FPGA board. In order to implement more than one cavity models in the given board (e.g. vector-sum control case), the multiplication algorithms need to be further optimized to save the hardware resources.

In this study, the simulator performance was mainly verified with cavities in KEK-STF and KEK-cERL. In what follows, the simulators in Fig. 11 and Fig. 12 are mainly based on the STF Phase-2 (STF2) nine-cell superconducting cavities operated in pulse mode [8,9]. Figure 13 and 14 are mainly based on beam experiment at STF [7]. Further, Figure 15 and 16 are mainly based on the nine-cell cavities in the cERL main linac operated in continuous wave $(\mathrm{CW})$ mode [12]. Figures 17-19 are based on the two-cell cavities in the cERL injector [10,12]. The LLRF and cavity 


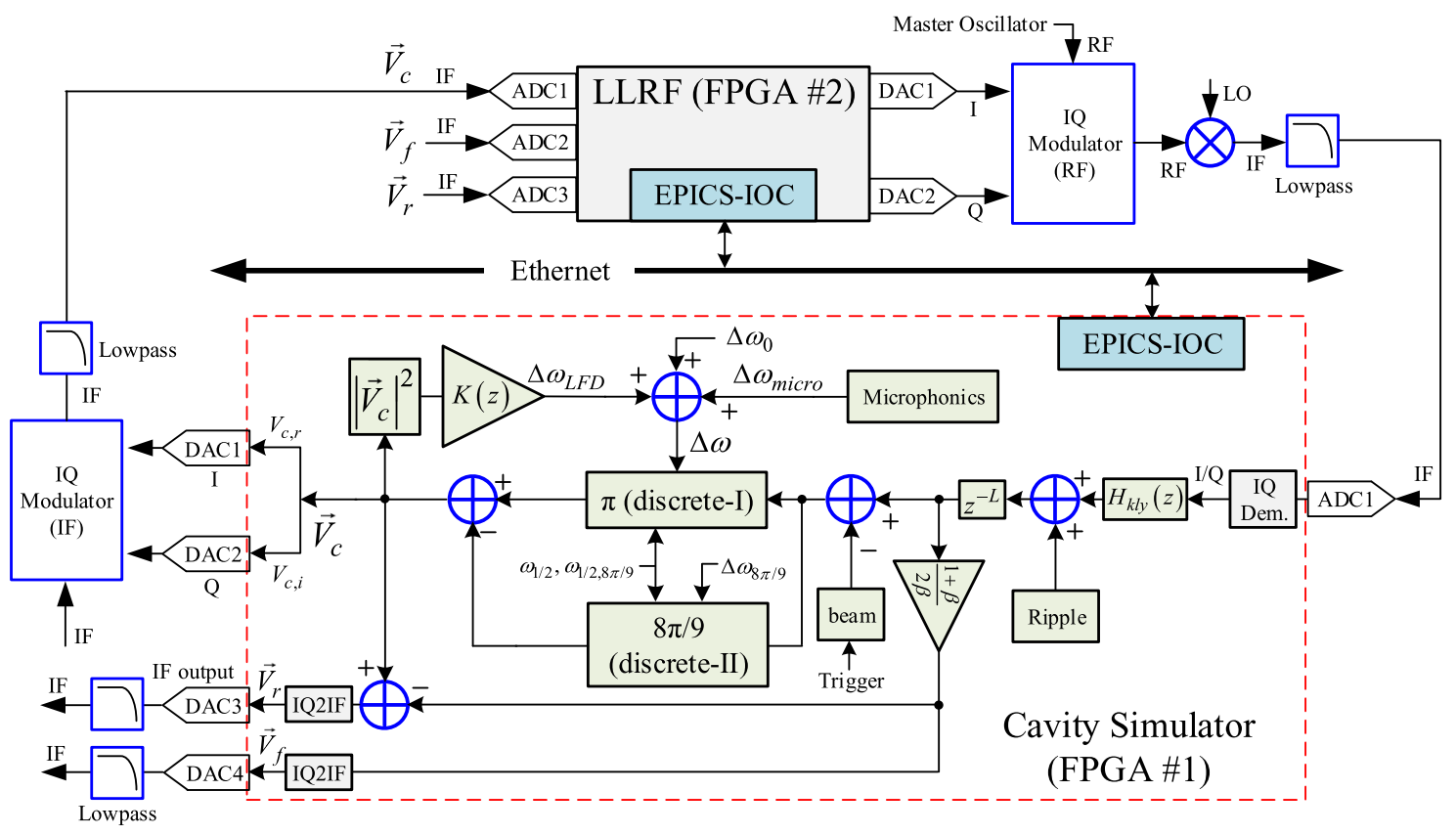

FIG. 10. Cavity simulator-based test bench for LLRF system. The cavity simulator model is implemented in FPGA \#1 (indicated by red block). Noted that only the ADCs and DACs in use are plotted in the figure.

parameters, and corresponding beam conditions for each figure are listed in Table II. To reject the $8 \pi / 9$ mode, a 4 th order IIR filter with $250 \mathrm{kHz}$ bandwidth was adopted in some experiments [24,25].

Figure 11 compares the measurement of the cavity pickup signal, incident signal $\left(P_{f}\right)$, and reflected signal $\left(P_{r}\right)$ from the KEK-STF2 nine-cell cavity (red) and that from the cavity simulator (blue) under "FF + FB" operation. It should be mentioned that in this paper the FF controller is a constant table (or value). The accelerating field is approximately $25 \mathrm{MV} / \mathrm{m}$. The results show that the signals of the rf pulse are in good agreement.

The LFD can be calibrated by the equation [26]

$$
\Delta \omega_{\mathrm{LFD}}=\frac{d \phi}{d t}-\frac{\omega_{1 / 2}(|\vec{u}|) \sin (\theta-\phi)}{\left|\vec{V}_{c}\right|}
$$

where $\phi$ and $\theta$ represent the phase of the cavity pick-up, $\vec{V}_{c}$, and the cavity incident voltage $\vec{V}_{f}$, respectively. The vector

TABLE I. Available and used $\mu$ TCA FPGA resources.

\begin{tabular}{|c|c|c|}
\hline Item & $\begin{array}{c}\text { Total } \\
\text { logicelements }\end{array}$ & $\begin{array}{l}\text { Hardware } \\
\text { multipliers } \\
\text { (DSP48Es) }\end{array}$ \\
\hline Available in $\mu$ TCA board & 44800 & 128 \\
\hline Cavity Simulator in Fig. 10 & 27676 & 95 \\
\hline $\begin{array}{l}\text { Electrical and mechanical Model } \\
\text { (Fig. 4) }\end{array}$ & 5410 & 48 \\
\hline
\end{tabular}

$\vec{u}$ is approximately equal to $2 \vec{V}_{f}$ in the case of a superconducting cavity.

Figure 12 compares the LFD measured for the KEK-STF2 nine-cell cavity (red) and that obtained from the presented cavity simulator (blue). The results are in good agreement, especially in terms of the filling time and the flat time of the rf pulse.

As shown in Fig. 13, the beam-loading effects are demonstrated by comparing the cavity pick-up signal
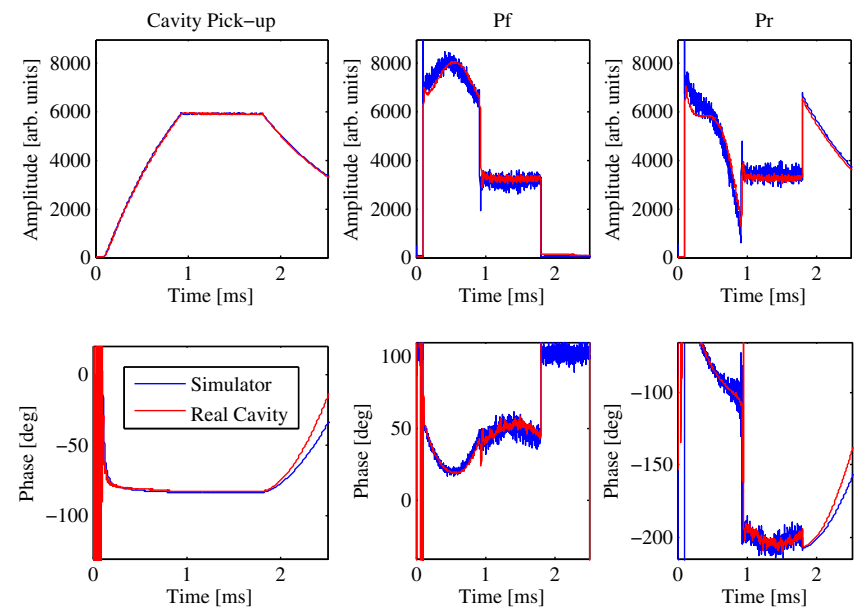

FIG. 11. Comparison of pick up, incident, and reflected cavity signals measurement based on cavity simulator (blue) and real STF nine-cell cavity (red) under "FF + FB" operation. The parameters of the cavity and mechanical models were mainly selected based on one of the nine-cell cavities in the STF2 $\left(Q_{L} \approx 4.8 \times 10^{6}, E_{\mathrm{acc}} \approx 25 \mathrm{MV} / \mathrm{m}\right)$. 


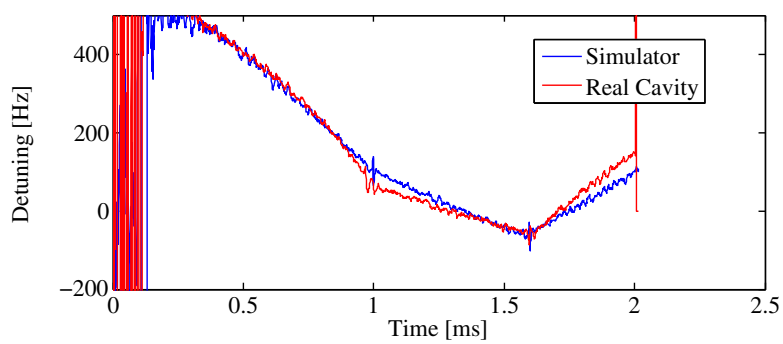

FIG. 12. Comparison of LFD between measurement based on cavity simulator (blue) and real STF2 nine-cell cavity (red).
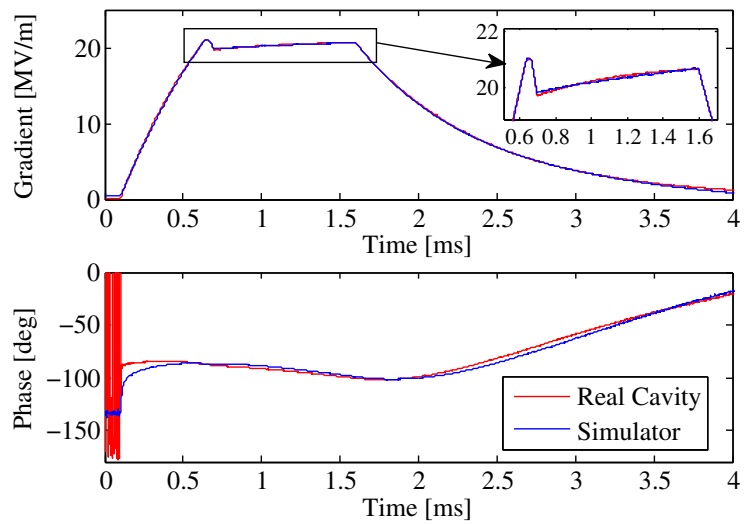

FIG. 13. Comparison of beam-loading effects between measurement based on cavity simulator (blue) and real STF nine-cell cavity (red).

between the cavity simulator and the real STF cavity. After injecting an on-crest beam of $31 \mu \mathrm{s}$ and $9.9 \mathrm{~mA}$, the beaminduced gradient drop can be easily observed in the amplitude of the cavity pick-up.

The rf phase for on-crest beam acceleration can be determined by a phase scan experiment. The beam induced changes in the cavity gradient is [7]

$$
\Delta V_{\text {ind }}=\pi \frac{r}{Q} f_{0} I_{b} \cos (\phi) \Delta t
$$

where $f_{0}$ is the resonance frequency, $\frac{r}{Q}$ the shunt impedance, $\phi$ the phase difference between of and beam current, and $\Delta t$ the beam transient time. If the phase difference is $-180^{\circ}$, the maximal cavity gradient drop will be observed as shown in Fig. 13. By performing the phase scan during beam operation, the rf phase for the on-crest beam acceleration can be determined. The results can be found in Fig. 14 where the beam induced gradient drop versus the rf phase is plotted. The rf phase for the on-crest beam acceleration was then determined by a sine-function fit.

The performance of the simulator in $\mathrm{CW}$ mode operation was mainly tested based on the parameters of KEK-cERL nine-cell and two-cell superconducting cavities.

In order to test the $8 \pi / 9$ mode of the cavity simulator, the detuning $\Delta \omega_{8 \pi / 9}$ was set to $1.72 \times 10^{6} \cdot 2 \pi \mathrm{rad} / \mathrm{s}$ (close to

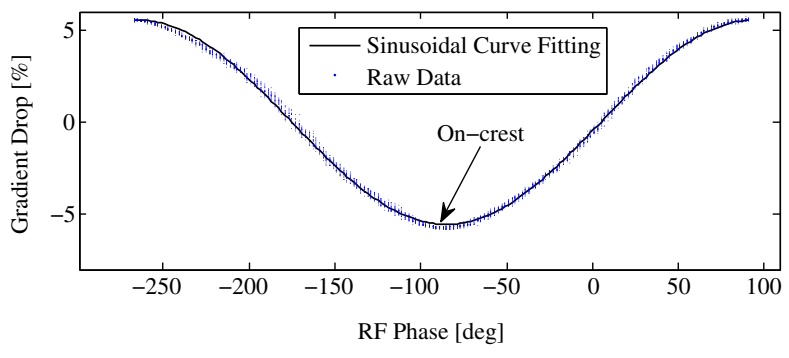

FIG. 14. Beam-induced change in the cavity gradient corrected by the gradient drop [\%] versus rf phase [deg] for the cavity at STF. The arrow indicates the on-crest beam acceleration.

the value for one of the cERL cavities) and then FB operation of the LLRF system was conducted. Figure 15 shows oscillations in case of FB operation due to the existence of the $8 \pi / 9$ mode.

As shown in Fig. 16, the microphonics model was confirmed by comparing the microphonics spectrum between the simulator (blue) and the second nine-cell superconducting cavity $\left(Q_{L} \approx 10 \times 10^{6}\right)$ in the cERL main linac (red). The results for FF and FB operation show that the main frequency components in the simulator and real cavity are similar.

The ripple model was validated by comparing the ripple waveform of the simulator and the cavities in the cERL injector, in which the klystron is used. The ripple frequency was set to approximately $300 \mathrm{~Hz}$, identical to the measured power-supply ripples. The results for the FF and FB operation in Fig. 17 show that the fluctuations caused by the ripples (and microphonics) in both the simulator and real cavity appear similar.
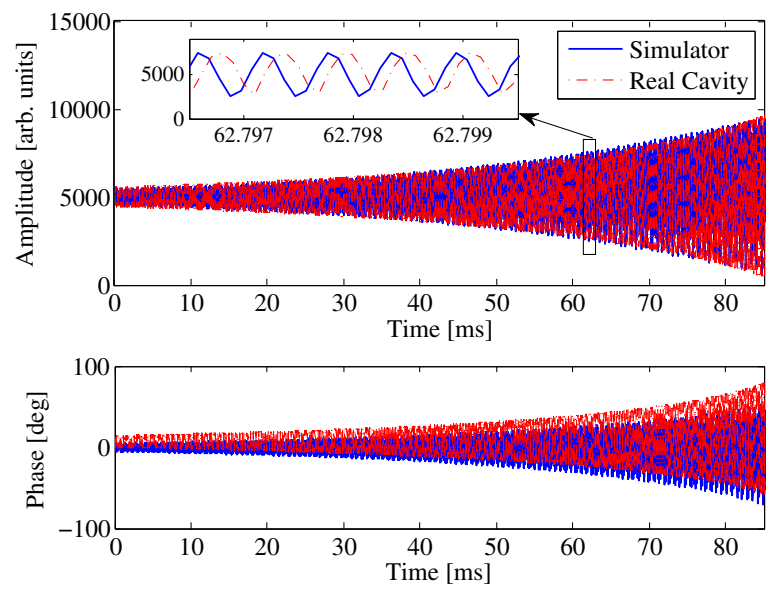

FIG. 15. Comparison of oscillations in cavity pickup signal caused by the $8 \pi / 9$ mode measured from cavity simulator (blue) and real cERL nine-cell cavity (red). The oscillation frequency in the figure is approximately $1.72 \mathrm{MHz}$ (closed to the value for one of the cavity in the main linac of cERL). Algorithms to generate the $8 \pi / 9$ mode were developed based on the difference equations in (14). 


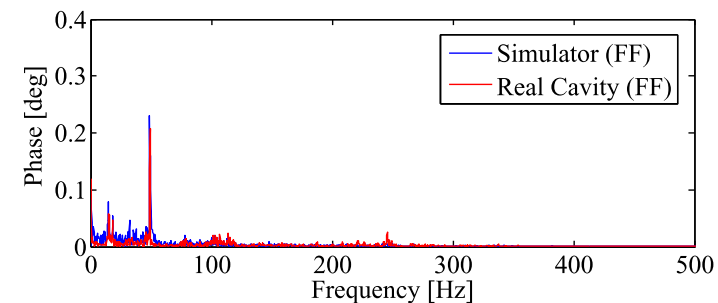

(a)

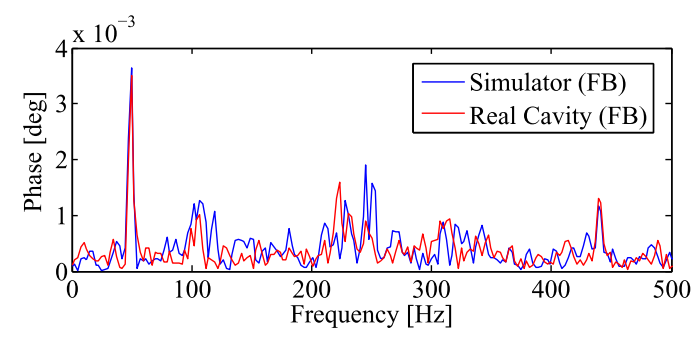

(b)

FIG. 16. Comparison of microphonics measured from cavity simulator-based LLRF system (blue) and LLRF system from cERL main linac (red) under FF operation. The cavity parameters were selected based on the cERL nine-cell cavity in the mainlinac $\left(Q_{L} \approx 10 \times 10^{6}\right)$. (a) FF and (b) FB control.

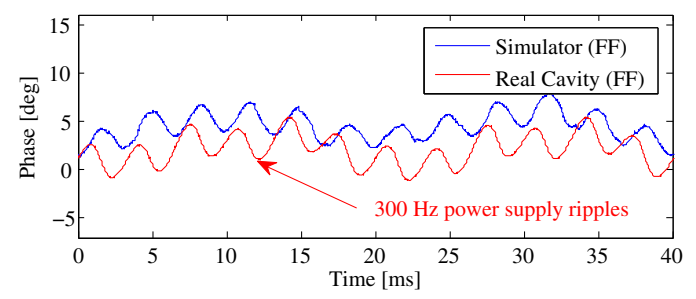

(a)

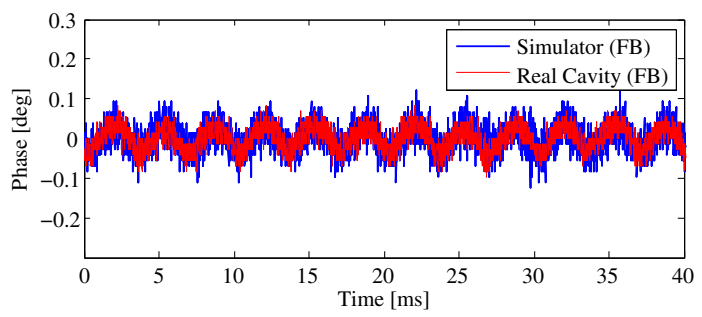

(b)

FIG. 17. Comparison of power-supply ripples measured based on cavity simulator (blue) and real cERL two-cell cavity (red) under FF operation. The ripple frequency was set to $300 \mathrm{~Hz}$, close to the real case. (a) FF and (b) FB control.

For the cERL beam commissioning, we have operated approximately beam in burst mode $(5 \mathrm{~Hz})$, as shown in Fig. $18[12,15]$. We adopted a trapezoid pulse to simplify the real beam current. Figure 19 compares the beamloading measured based on the cERL injector cavity (red) and the cavity simulator (blue). The results for the two are in good agreement after proper scaling.

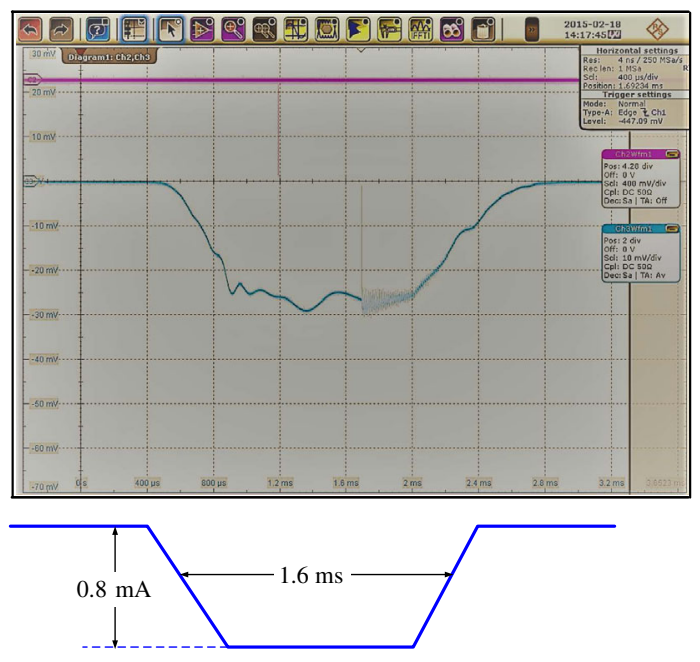

FIG. 18. (Upper) 1.6-ms and 800- $\mu \mathrm{A}$ beam current measured by oscilloscope and (lower) simulated current used in simulator.
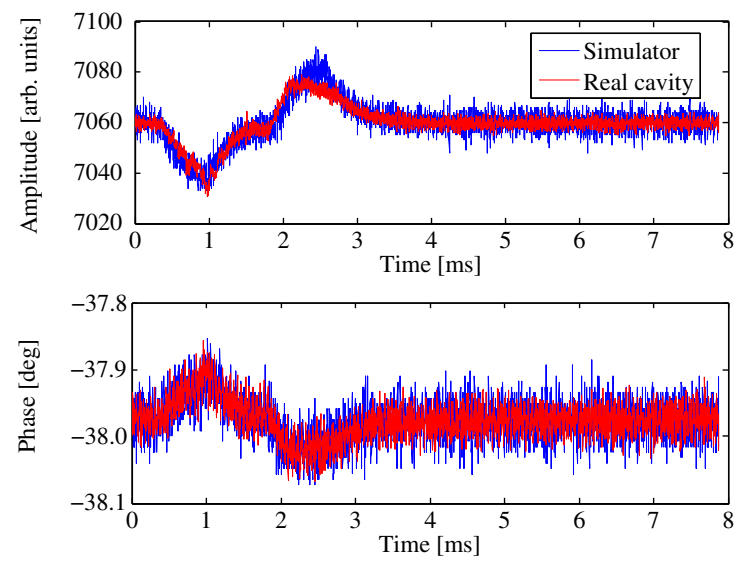

FIG. 19. Comparison of beam-loading effects for cavity simulator-based LLRF system (blue) and LLRF system for cERL injector (red) under FB operation. The cavity parameters were selected based on the cERL nine-cell cavity in the injector $\left(Q_{L} \approx 1.2 \times 10^{6}\right)$ and the $\mathrm{P}$ gain for feedback was approximately 50 .

\section{LLRF APPLICATIONS BASED ON CAVITY SIMULATOR}

It is very convenient to design and optimize the LLRF system in the presence of the cavity simulator. For a specified LLRF application, after the design and development process, the system was always confirmed in the cavity simulator first, and then modified if bugs were detected. Using this approach, making the real rf source and beam operation are unnecessary, we can conserve precious machine time and increase the development efficiency.

For the cERL and STF, some cavity simulator-based LLRF applications are presented below. 
TABLE II. LLRF and cavity parameters and corresponding beam conditions for Figs. 11-19.

\begin{tabular}{|c|c|c|c|c|c|c|c|c|c|}
\hline \multirow[b]{2}{*}{ Figure } & \multirow[b]{2}{*}{ Confirmation } & \multirow[b]{2}{*}{ Facility } & \multirow[b]{2}{*}{ Mode } & \multirow[b]{2}{*}{$\begin{array}{l}\text { Beam Conditions } \\
\qquad\left(I_{b}, \phi_{b}\right)\end{array}$} & \multicolumn{3}{|c|}{ Cavity Parameters } & \multicolumn{2}{|c|}{ LLRF Parameters } \\
\hline & & & & & $E_{\text {acc }}$ or $V_{c}$ & Loaded Q & $\begin{array}{l}\text { Cavity } \\
\text { type }\end{array}$ & $\begin{array}{l}\text { Control } \\
\text { method }\end{array}$ & Filter \\
\hline 11 & Normal operation & STF-2 & Pulse & W/o beam & $25 \mathrm{MV} / \mathrm{m}$ & $4.8 \times 10^{6}$ & Nine-cell & $\mathrm{FF}+\mathrm{FB}$ & IIR(4th) \\
\hline 12 & LFD effects & STF-2 & Pulse & W/o beam & $30 \mathrm{MV} / \mathrm{m}$ & $4.8 \times 10^{6}$ & Nine-cell & FF & IIR(4th) \\
\hline 13,14 & Beam-loading effects & STF & Pulse & $\left(9.9 \mathrm{~mA}, 0^{\circ}\right)$ & $21 \mathrm{MV} / \mathrm{m}$ & $3.2 \times 10^{6}$ & Nine-cell & $\mathrm{FF}$ & W/o filter \\
\hline 15 & Parasitic mode effects & cERL & $\mathrm{CW}$ & W/o beam & $2.5 \mathrm{MV}$ & $14 \times 10^{6}$ & Nine-cell & FB & W/o filter \\
\hline 16 & Microphonics effects & cERL & $\mathrm{CW}$ & $\left(6.5 \mu \mathrm{A}, 0^{\circ}\right)$ & $8.5 \mathrm{MV}$ & $10 \times 10^{6}$ & Nine-cell & $\begin{array}{c}\mathrm{FF}(\mathrm{a}) \text { and } \\
\mathrm{FB}(\mathrm{b})\end{array}$ & IIR(4th) \\
\hline 17 & PS ripples effects & cERL & $\mathrm{CW}$ & $\left(6.5 \mu \mathrm{A}, 0^{\circ}\right)$ & $0.65 \mathrm{MV}$ & $0.5 \times 10^{6}$ & Two-cell & $\begin{array}{c}\mathrm{FF}(\mathrm{a}) \text { and } \\
\mathrm{FB}(\mathrm{b})\end{array}$ & $\operatorname{IIR}(1 \mathrm{st})$ \\
\hline 18,19 & Beam-loading effects & cERL & Burst & $\left(0.8 \mathrm{~mA}, 0^{\circ}\right)$ & $0.7 \mathrm{MV}$ & $1.2 \times 10^{6}$ & Two-cell & FB & IIR(1st) \\
\hline
\end{tabular}

\section{A. Suppression of $\mathbf{8} \pi / \mathbf{9}$ mode}

In order to suppress the $8 \pi / 9$ modes in the nine-cell cavities at KEK-cERL and KEK-STF, we have developed several kinds of digital filters, including a first-order IIR filter, a fourth-order conjugate-pole IIR filter, and a notch filter [24]. We have applied these three filters in the LLRF system, first demonstrating them on the cavity simulator.

Figure 20 compares the close loop performance with and without a filter. First, we feedbacked the system without a filter and gradually increased the $\mathrm{P}$ gains to a very low value (less than 20). However, system oscillation was observed (indicated by the oscillation in blue) due to the existence of the $8 \pi / 9$ mode. In the next step, we activated the filter and the oscillations disappeared (indicated by the waveform in red), even if the gain was larger than 300 .

The results show that both the notch filter and the fourthorder conjugate-pole filter perform well with high gain operation. We finally selected the latter because of its benefits with regard to the FPGA resource cost [24].

After confirming the filter in the cavity simulator-based test bench, we applied it in real accelerators such as cERL and STF, where the filter was found to perform well $[24,25]$.
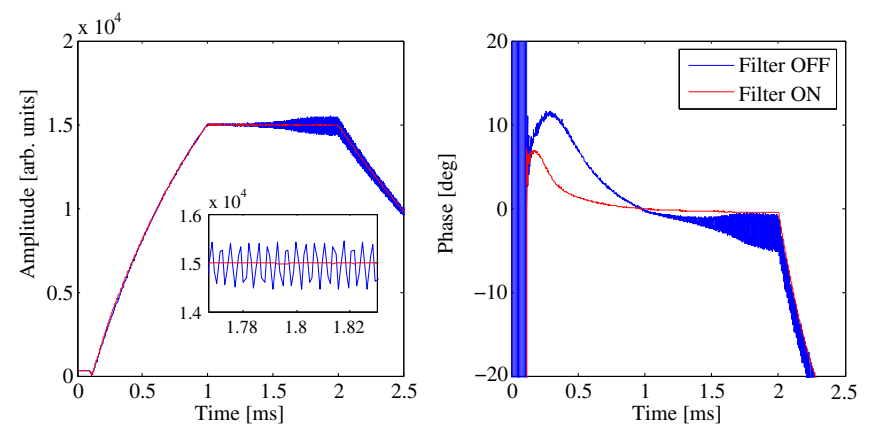

FIG. 20. Closed-loop operation based on cavity simulator with and without filter. In the without filter case (blue line), even a very low gain $(\mathrm{P}$ gain $\approx 20$ ) resulted in system oscillation, because of the existence of the $8 \pi / 9$ mode. In the with-filter case, the system was stable, even with high $\mathrm{P}$ gain up to 300 .

\section{B. Disturbance observer-based control}

As mentioned above, disturbances such as microphonics, power-supply ripples, and beam-loading limit the LLRF system performance. The traditional PI control is usually insufficient to suppress them. In order to improve the system performance, a disturbance observer-based (DOB) control method has previously been applied in the KEK-cERL to reject the disturbances in the LLRF system [13-15]. Following completion of the DOB controller design, we first demonstrated this approach on the cavity simulator.

A simulator-based experiment aiming to compensate for the beam-loading in the burst mode operation during the cERL beam commissioning was performed. At first, we tried to operate the LLRF system with PI feedback control; and we have optimized the PI gains to have a best performance. The cavity parameters are mainly based on the two-cell cavcity in the cERL injector. In the next step, we injected the 1.6-ms and 800-mA beam current in our simulator, as mentioned above, a trapezoid pulse was adopted to simplify the real beam current (see Fig. 18). The beam effects were obvious in the cavity pick-up especially for the amplitude, as shown by the blue waveforms in Fig. 21. Finally, we switched on the DOB controller, and the system is then operated under "PI + DOB" control. It is clear to see that the beam-loading

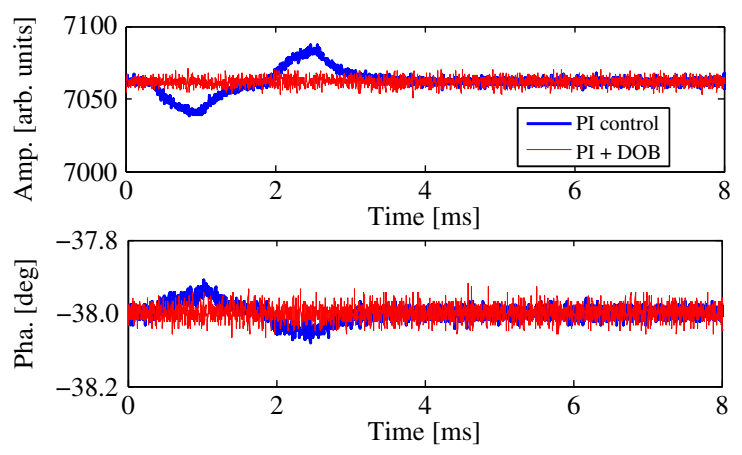

FIG. 21. Measured cavity pick-up signal on cavity simulator in presence of $0.8-\mathrm{mA}$ beams. The blue and red waveforms represent the PI and "PI + DOB"-based control, respectively. 
effects were greatly improved by the applied DOB control approach, as shown by the red waveforms in Fig. 21. After the demonstration in the cavity simulator, we successfully applied and confirmed this approach in real accelerators at cERL and STF [13-15].

\section{SUMMARY AND OUTLOOK}

In order to test the LLRF system and to demonstrate LLRF approaches before application in the field, we have designed an FPGA-based cavity simulator including cavity and klystron models, along with several main disturbance models. Experiments based on the developed cavity simulator show that the dynamic behavior of the simulator is very close to the behavior of the real cavity system. LLRF applications such as suppression of the $8 \pi / 9$ mode, and DOB control were first demonstrated based on the cavity simulator and then confirmed successfully at KEK accelerators.

It should be mentioned that the presented simulator is mainly designed to deal with the field control issue. The resonance control in $\mathrm{CW}$ mode is not taken in to account so far. For further study, we will implement some second order models in FPGA to simulate the behavior of the microphonics; therefore, the microphonics compensation algorithms can be studied based on the simulator. Furthermore, non-linear characteristics of the klystron will be considered and simulated in the klystron model of the simulator, thus a more realistic test environment for the rf system can be realized. In addition, a model to simulate the temperature drift will be integrated in the simulator, and the effect of the drift can be then learned in the test bench.

[1] J. K. Keung et al., in Proceedings of Particle Accelerator Conference (PAC07), New Mexico, USA (JACoW, New Mexico, USA, 2007), MOPAS086, p. 632.

[2] P. Orel, and U. Mavrič, Analog cavity simulator, Nucl. Instrum. Methods Phys. Res., Sect. A 729, 241 (2013).

[3] T. Czarski, K. T. Pozniak, R. S. Romaniuk, and S. Simrock, TESLA cavity modeling and digital implementation in FPGA technology for control system development, Nucl. Instrum. Methods Phys. Res., Sect. A 548, 283 (2005).

[4] A. Grassellino et al., in Proceedings of Particle Accelerator Conference (PAC07), New Mexico, USA (JACoW, New Mexico, USA, 2007).

[5] Z. Geng et al., Study of the digital LLRF system for STF, High Energy Phys. Nucl. Phys. 31, 774 (2007).

[6] H. Hayano et al., in Proceedings of the 8th Annual Meeting of the Particle Accelerator Society of Japan, Tsukuba, Japan (PASJ, Japan, 2011), p. 893.

[7] M. Omet, H. Hayano, A. Kuramoto, T. Matsumoto, S. Michizono, T. Miura, and F. Qiu, High-gradient nearquench-limit operation of superconducting Tesla-type cavities in scope of the International Linear Collider, Phys. Rev. ST Accel. Beams 17, 072003 (2014).
[8] Y. Yamamoto et al., in Proceedings of the 7th International Particle Accelerator Conference (IPAC2015), Busan, Korea (JACoW, Busan, Korea, 2016), WEPMB017, p. 2158.

[9] S. B. Wibowo, T. Matsumoto, S. Michizono, T. Miura, F. Qiu, and N. Liu, Digital low level rf control system with four different intermediate frequencies for the International Linear Collider, Phys. Rev. Accel. Beams 20, 093501 (2017).

[10] S. Sakanaka et al., in Proceedings of 53rd ICFA Advanced Beam Dynamics Workshop on Energy Recovery Linacs (ERL 2013), Novosibirsk, Russia (JACoW, Novosibirsk, Russia, 2013), WG102, p. 16.

[11] N. Nakamura et al., in Proceedings of the 5th International Particle Accelerator Conference (IPAC2014), Dresden, Germany (JACoW, Dresden, Germany, 2014), MOPRO110, p. 353.

[12] M. Akemoto et al., Construction and commissioning of compact energy-recovery linac at KEK, Nucl. Instrum. Methods Phys. Res., Sect. A 877, 197 (2018).

[13] F. Qiu et al., in Proceedings of the 6th International Particle Accelerator Conference (IPAC2015), Richmond, USA (JACoW, Richmond, USA, 2015), WEPMA054, p. 2895.

[14] F. Qiu et al., in Proceedings of the 56th ICFA Advanced Beam Dynamics Workshop on Energy Recovery Linacs (ERL2015), New York, USA (JACoW, New York, USA, 2015), WEIDLH2066, p. 84.

[15] F. Qiu, S. Michizono, T. Miura, T. Matsumoto, M. Omet, and B. W. Sigit, Application of disturbance observer-based control in low-level radio-frequency system in a compact energy recovery linac at KEK, Phys. Rev. ST Accel. Beams 18, 092801 (2015).

[16] T. Schilcher, Ph.D. thesis, Universitt Hamburg, 1998.

[17] E. Vogel, High gain proportional rf control stability at TESLA cavities, Phys. Rev. ST Accel. Beams 10, 052001 (2007).

[18] M. U. Liepe, Ph.D. thesis, Universitt Hamburg, 2001.

[19] M. Omet et al., FPGA-based klystron linearization implementations in scope of ILC, Nucl. Instrum. Methods Phys. Res., Sect. A 780, 1 (2015).

[20] M. Hara et al., A ripple effect of a klystron power supply on synchrotron oscillation, Part. Accel. 59, 143 (1998).

[21] A. Neumann, W. Anders, O. Kugeler, and J. Knobloch, Analysis and active compensation of microphonics in continuous wave narrow-bandwidth superconducting cavities, Phys. Rev. ST Accel. Beams 13, 082001 (2010).

[22] A. Neumann, Ph.D. thesis, Humboldt-Universitt zu Berlin, 2008.

[23] T. H. Kandil, Adaptive feedforward cancellation of sinusoidal disturbances in superconducting RF cavities, Nucl. Instrum. Methods Phys. Res., Sect. A 550, 514 (2005).

[24] F. Qiu et al., in Proceedings of the 27th Linear Accelerator Conference (LINAC14), Geneva, Switzerland (JACoW, Geneva, Switzerland, 2014), MOPP074, p. 227.

[25] B. W. Sigit et al., in Proceedings of the 8th International Particle Accelerator Conference (IPAC17), Copenhagen, Denmark (JACoW, Copenhagen, Denmark, 2017), THPAB116, p. 3992.

[26] A. Brandt, Ph.D. thesis. Universitat Hamburg, 2007. 